\title{
Routing of Traffic Sensors in Intelligent Transportation System
}

\author{
Swathi Shastry and T.R. Naveenkumar
}

\begin{abstract}
As country develops, the application of technology in each and every field increases to fulfill the demand of people. The application of technology in transportation system is called Intelligent Transportation System (ITS) which has more demand in today's world for traffic management. Vehicular Ad hoc Network (VANET) is one of the technologies used in Intelligent Transportation System. In Vehicular Ad hoc Network temporary network is formed within the vehicles or vehicle to traffic infrastructure which has sensors within it for communication. The temporary network establishes and ends after exchanging the required information. Numerous technologies have been deployed to assist and manage transportation. But recent concerted efforts in academia and industry point to a paradigm shift in intelligent transportation systems. Vehicles will carry computing and communication platforms, and will have enhanced sensing capabilities. They will enable new versatile systems that enhance transportation safety and efficiency and will provide infotainment. The communication between vehicles can be set to avoid accidents by identifying it's distance between the vehicles, number of vehicle in the specified range.
\end{abstract}

\section{INTRODUCTION}

\section{A. Intelligent Transportation System}

UUMEROUS technologies have been deployed to assist 1 and manage transportation. Vehicles will carry computing and communication platforms, and will have enhanced sensing capabilities. They will enable new versatile systems that enhance transportation safety and efficiency and will provide infotainment. VANET is necessary for transportation system to obtain real time traffic information like origin-destination trips, route flow, link flow, travel time estimation etc. In VANET, sensors collect the information of vehicles to provide transportation services like travel and traffic management, emergency management, electronic payment etc. Vehicular Ad hoc Network (VANET) is a type of Mobile Ad hoc Network (MANET) used in Intelligent Transportation System to obtain traffic information. VANET is a network formed by moving vehicles with no infrastructure which is formed instantaneously.

Swathi Shastry, Assistant Professor, Department of CSE, KLS, VDRIT, Haliyal.E-mail:swathishastry9688@gmail.com

T.R. Naveenkumar, Assistant Professor, Department of CSE, KLS, VDRIT, Haliyal.E-mail:trnaveenkumar75@gmail.com

DOI:10.9756/BIJSESC.8252

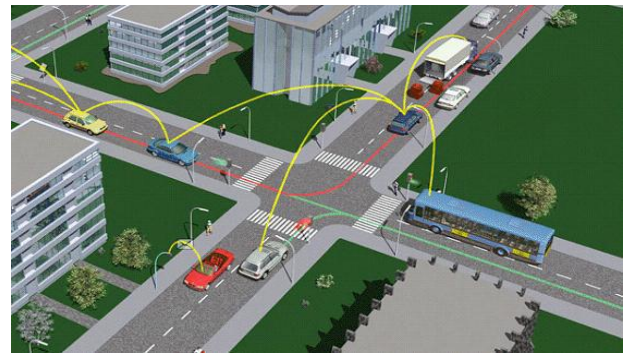

Figure 1: Transportation System Using Mobile Wireless Sensor Network

The travel time estimation is the application used in logistic departments to deliver goods to customers on time. By using information of travel time estimation one can understand the condition of road, traffic congestion etc. and calculate the travel time for delivering particular goods. Routing protocols are used to transfer data in a network between entities. In vehicular Ad hoc network (VANET) routing is challenging task because of dynamic change in speed of vehicles. VANET form a temporary network which consists of sensors in moving vehicles. The sensors in vehicles communicate within themselves and exchange of information is possible. The node to infrastructure communication is possible with cellular network. Vehicular Ad-Hoc Networks (VANETs) is the type of MANETs (Mobile Ad-Hoc Networks) which belongs to Wireless Ad-Hoc Networks. It is a technology which integrates Ad-Hoc networks, wireless LAN, and cellular technology to achieve intelligent vehicular communications (IVC) and roadside to vehicle communication (RVC).The aim of VANETs is to provide safety and comfort for travelers. This network tends to operate without any infrastructure. Each vehicle equipped with VANET device will be a node in the Ad-Hoc network and can receive and relay others messages through the wireless network.

\section{B. Some Unique Characteristics of VANETs are}

a) Potentially high number of nodes: A large number of vehicles take part in communication in VANETs. Inconsideration of Road side units, (RSU), VANETs needs to be scalable with high number of nodes.

b) Self-organized and distributed network: Since vehicles move randomly, the network is self-organized and distributed network (work is done by one node and it is shared among other nodes).

c) No requirement of Infrastructure: The entities are not permanently connected and do not require any Base station. High mobility and frequency topology changes: Vehicles move with considerably high speed and the network frequently changes. Hence, the 
duration for the exchange of date packets is rather small.

d) High application requirement on data delivery: Important application is traffic safety to avoid road accidents and other non-safety applications.

e) No confidentiality of safety information: Here, safety information is passed from one vehicle to another vehicle. Hence there is no confidentiality.

f) Privacy: Little sensitive information like driver's identity, speed, position and mobility pattern should be kept secret. However, when information is exchanged between vehicles, there is a possibility of this information to be spread. So privacy is an important factor in VANETs.

g) No concern about Power: It depends on the life of the vehicle which is not a concern in VANETs.

\section{LITERATURE REVIEW}

\section{A. Distributed Trust \& Time Management Strategy in VANETs}

Distributed trust management in VANET is a challenging task due to the lack of infrastructure, openness of wireless links and the usually highly dynamic network topology. The communication between vehicles is used for safety, comfort and entertainment (convenience). The performance of communication depends on how better the routing takes place in the network. Routing of data depends on the routing protocols being used in network. To overcome security threats of VANETs, each broadcast message must contains some authentication information to help receiver to validate the authenticity of the information[1].

\section{B. A RSU-Aided Scheme for Data-centric Trust Establishment in VANETs}

A Roadside-unit Aided Trust Establishment scheme (RATE) that aims to execute data-centric trust establishment in VANET efficiently1. Compared to existing trust establishment approaches, RATE is completely data-centric, making RATE appropriate for rapidly changing environment. In addition, through the employment of ant colony optimization algorithm, RATE has ability to integrate direct observed data with feedback information when evaluating the trustworthiness of data, eventually improves the accuracy of evaluation result. Lastly, RATE decouples data consuming and data-providing entities, thus enables vehicles to mitigate the attacks launched by malicious nodes through directlylinked communications. Simulation shows that RATE can effectively analyze data provided in VANET and correctly establish trust on data.[3]

\section{Routing of Traffic Sensors in Intelligent Transportation System}

Vehicular Ad hoc Network (VANET) is a type of Mobile Ad hoc Network (MANET) used in Intelligent Transportation System to obtain traffic information. VANET is a network formed by moving vehicles with no infrastructure which is formed instantaneously. VANET architecture can be divided into three types WLAN network, Ad Hoc network and Hybrid network. Sensors are another important device which consists of basic elements like microcontroller, radio transceiver and energy source. The travel time estimation is the application used in logistic departments to deliver goods to customers on time. By using information of travel time estimation one can understand the condition of road, traffic congestion etc. and calculate the travel time for delivering particular goods. Routing protocols are used to transfer data in a network between entities. In vehicular Ad hoc network (VANET) routing is challenging task because of dynamic change in speed of vehicles. In highways the vehicles average speed is $65 \mathrm{~km} / \mathrm{h}$ i.e. $17 \mathrm{~m} / \mathrm{s}$, so the routing should be very quick in fraction of seconds. VANET form a temporary network which consists of sensors in moving vehicles. The sensors in vehicles communicate within themselves and exchange of information is possible. [3] The node to infrastructure communication is possible with cellular network. Due to the mobility of vehicles, the topology of network changes frequently, breakages of network links and no central control. So both topology based and position based routing are proposed previously. The AODV (Ad hoc On Demand Vector) protocol is a type of reactive protocol that forms the route whenever required. In route discovery, the route request packet is sent from source to the RSU which in turn sends it to central control unit which is maintained by transportation authority. The destination RSU receives the packet from Central control unit. One of the node is selected as relay node among all nodes, dynamically which establishes route and using shortest path sends the packets. Effective routing is most difficult task in mobile vehicles because of frequent change in topology. To achieve effective routing two stage heuristic hybrid algorithm is proposed. The two stage heuristic algorithm is based on particle swarm optimization and ant colony optimization. The ant colony optimization is upper level algorithm and particle swarm optimization follows the route produced by ant colony optimization. So that communication is more effective with shortest route. [2]

The objective of ambient intelligence is to create an intelligent daily space, which is immediately usable and integrated into our homes, our offices, our roads, our cars, and everywhere. This new concept must be invisible; it must blend in with our normal environment and must be present when we need it. One of the applications of this concept consists of providing our cars and roads with capabilities to make the road more secure (information about the traffic, accidents, dangers, possible detours, weather, etc.) and to make our time on the road more enjoyable (Internet access, network games, helping two people follow each other on the road, chat, etc.). These applications are typical examples of what we call an Intelligent Transportation System (ITS) whose goal is to improve security, efficiency and enjoyment in road transport through the use of new technologies for information and communication (NTIC).[5]

Traditional traffic management systems are based on centralized infrastructures where cameras and sensors implemented along the road collect information on density and traffic state and transmit this data to a central unit to process it and make appropriate decisions. This type of system is very costly in terms of deployment and is characterized by a long reaction time for processing and information transfer in a 
context wh this type of system. In addition, these devices placed on roads require periodic and expensive maintenance. Consequently, for large scale deployment of this type of system, important investment is required in the communication and sensor infrastructure. However, with the rapid development of wireless communication technologies, location and sensors, a new decentralized (or semi-centralized) architecture based on vehicle-to-vehicle communications $(\mathrm{V} 2 \mathrm{~V})$ has created a very real interest these last few years for car manufacturers, R\&D community and telecom operators. [3] This type of architecture relies on a distributed and autonomous system and is made up of the vehicles themselves without the support of a fixed infrastructure for data routing. In this case, we are talking about a vehicular ad hoc network (VANET), which is no more than a specific application of traditional mobile ad hoc networks (MANET) 1ere information transmission delay is vital and is extremely important in this type of system. In addition, these devices placed on roads require periodic and expensive maintenance. Consequently, for large scale deployment of this type of system, important investment is required in the communication and sensor infrastructure. However, with the rapid development of wireless communication technologies, location and sensors, a new decentralized (or semi-centralized) architecture based on vehicle-to-vehicle communications $(\mathrm{V} 2 \mathrm{~V})$ has created a very real interest these last few years for car manufacturers, R\&D community and telecom operators. This type of architecture relies on a distributed and autonomous system and is made up of the vehicles themselves without the support of a fixed infrastructure for data routing. In this case, we are talking about a vehicular ad hoc network (VANET), which is no more than a specific application of traditional mobile ad hoc networks (MANET). [8]

\section{PROPOSED SYSTEM}

We have simulated vehicle to vehicle communication to avoid accidents. This is very useful in heavy traffic and place where lots of accidents happen. This provides communication with other vehicles, distance between the vehicles, and number of vehicles in the communicating range. This is very useful in real time traffic management and is also trustworthy. Objective of this is that, Vehicles will carry computing and communication platforms, and will have enhanced sensing capabilities. They will enable new versatile systems that enhance transportation safety and efficiency and will provide infotainment. Few of services provided by vehicle to vehicle communication system application are:

1) Alert in case of accidents

2) Alert in case of abnormally slow traffic

3) Collaborative driving

4) High hot spot

5) Parking management

\section{SYSTEM DESIGN}

1. Concept of Operations: This document defines the problem, the project's goals, stakeholder needs and expectations, constraints, and the way the ITS system is required to operate and be maintained.
2. System Requirements: This document contains specifications of what the system is required to do, how well it is required to do it, and under what conditions. These requirements are based on the goals, stakeholder needs and expectations, constraints, and operation and maintenance requirements documented in the Concept of Operations.

3. System Verification Plan: This document describes how the agency will verify that the system being built meets the requirements in the System Requirements document. The agency will implement the System Verification Plan to ensure all system requirements are verified before it accepts the system.

4. System Validation Plan: This document describes how the agency will assess the system's performance against the goals, stakeholder needs and expectations, constraints, and operation and maintenance requirements documented in the Concept of Operations. The goal is for the agency to understand and review the strengths and weaknesses of the system and identify any new opportunities and needs if appropriate. The agency will implement the System Validation Plan after it accepts the system.

The systems engineering process contains a number of steps that are not included in a traditional project delivery process. The systems engineering process is often referred to as the "V" diagram shown in figure 2. An ITS project begins on the left side of the "V" and progresses down the left side and then up the right side. Then the project is evaluated by validating and verifying the elements on the right side of the "V" with the elements on the left side.

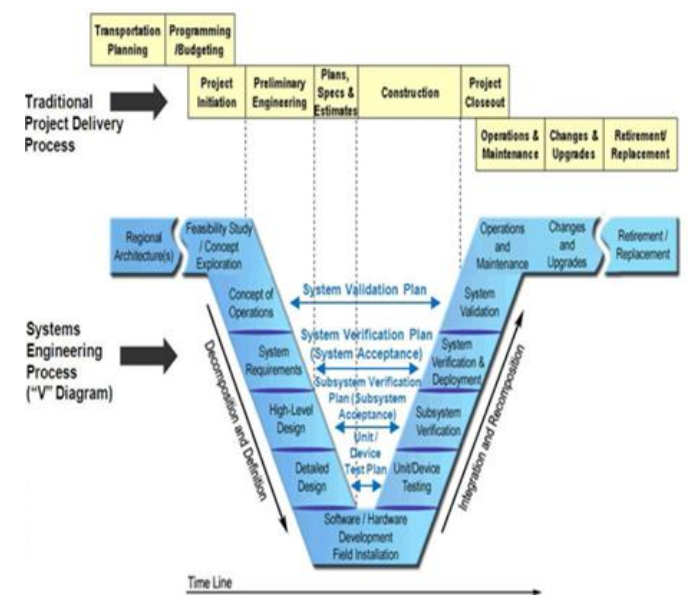

Figure 2: "V" Diagram

Vehicular communications in fig 3 can be classified into V2V and V2I. V2I further includes communication using cellular networks. Basically, Communication Models are as follows:

a) Vehicle to Vehicle (V2V): V2V involves direct communication between vehicles in their communication range. $\mathrm{V} 2 \mathrm{~V}$ is an automobile technology designed to allow automobiles to "talk" to each other. Vehicular ad-hoc network (VANET) is formed among vehicles for exchanging information e.g., safety information. 
b) Vehicle to infrastructure (V2I): Vehicle to fixed Infrastructure (V2I) in which each vehicle is commutate with a fixed road infrastructure i.e. Road Side Unit (RSU) through dedicated short range communication (DSRC), Wi-Fi, 3G or 4G.

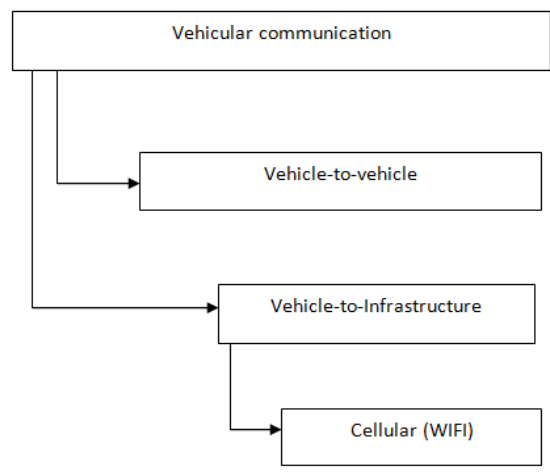

Figure 3: Vehicle Communication

\section{Use Case Diagram}

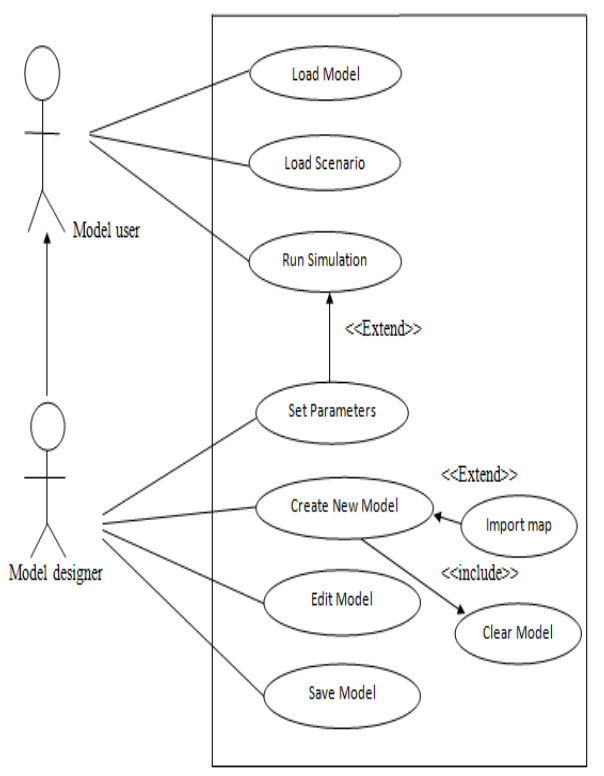

Figure 4: Use Case Diagram

\section{IMPLEMENTATION}

Figure 4 gives the working of VANET simulator, loading of map to VANET simulator, information about the vehicles, communication range of vehicle, shows vehicle id, speed of vehicle, known penalty, active vehicles, average speed etc. are displayed in process of simulation. We can load different map and vehicles according to the map. We can set how much number of vehicles to be loaded to the map. In simulation there will be notification given to vehicle so avoid accident.

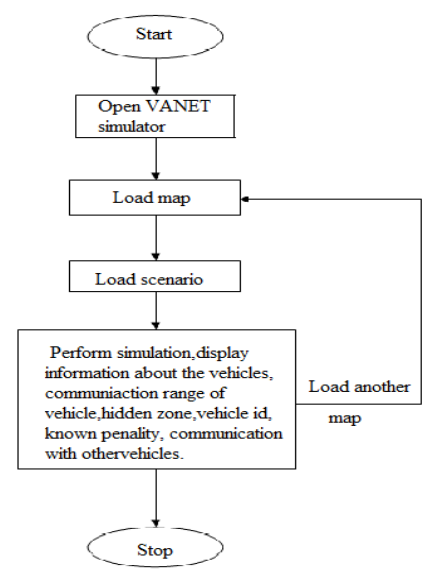

Figure 5: Flowchart for Working of VANET Simulator

VI. RESULTS

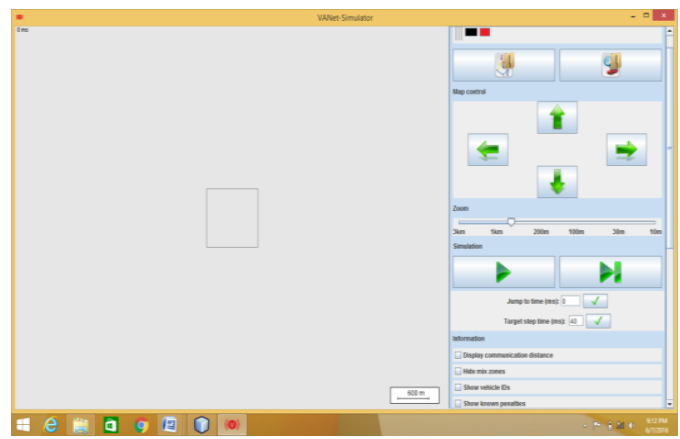

Figure 6: Open VANET Simulator to Load Map

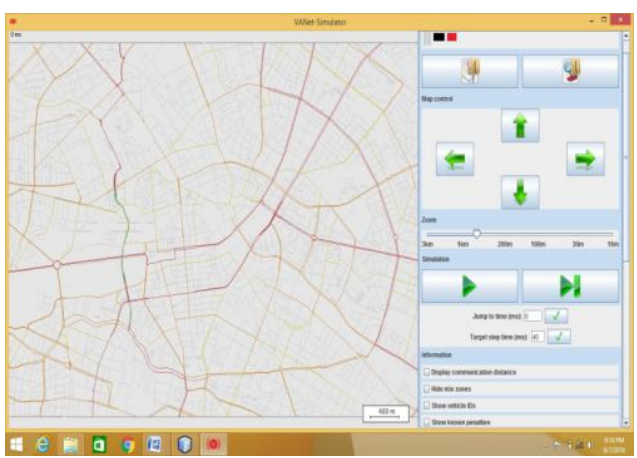

Figure 7: After Loading Map

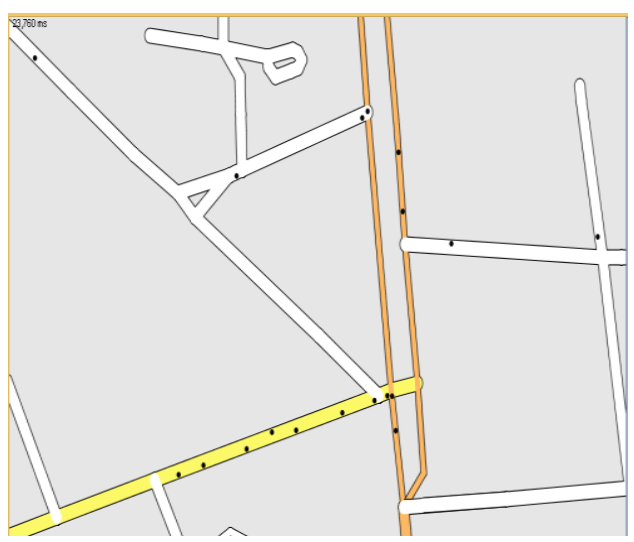

Figure 8: Loading of Scenario (i.e. Loading of Vehicles onto the Map) 


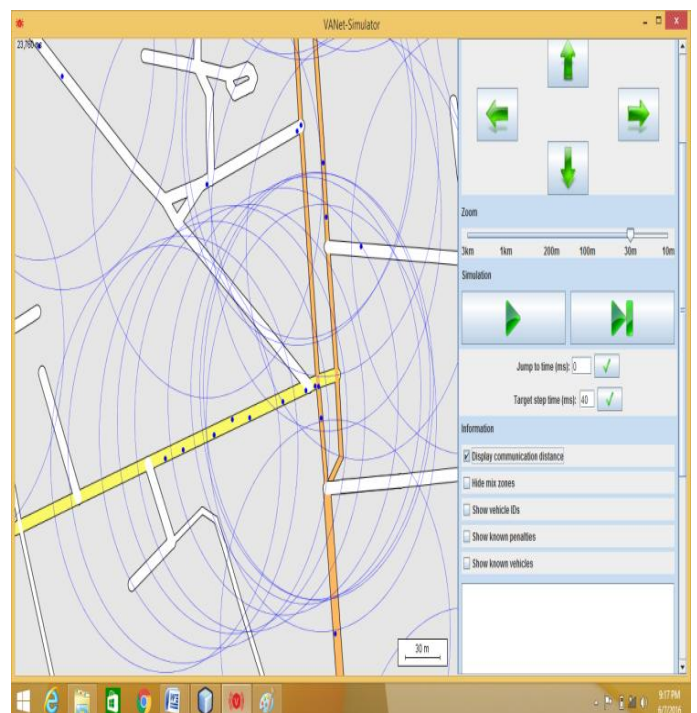

Figure 9: Communication Range of Each Vehicle

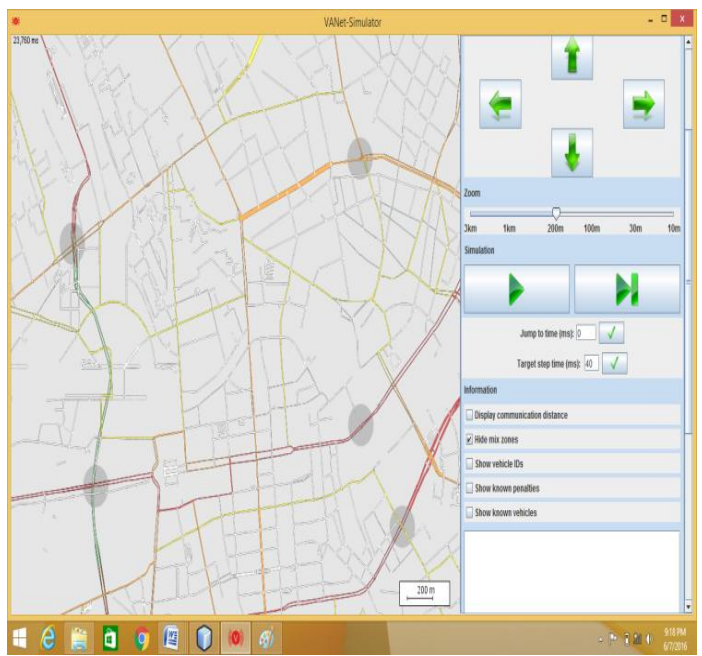

Figure 10: Hidden Mix Zone

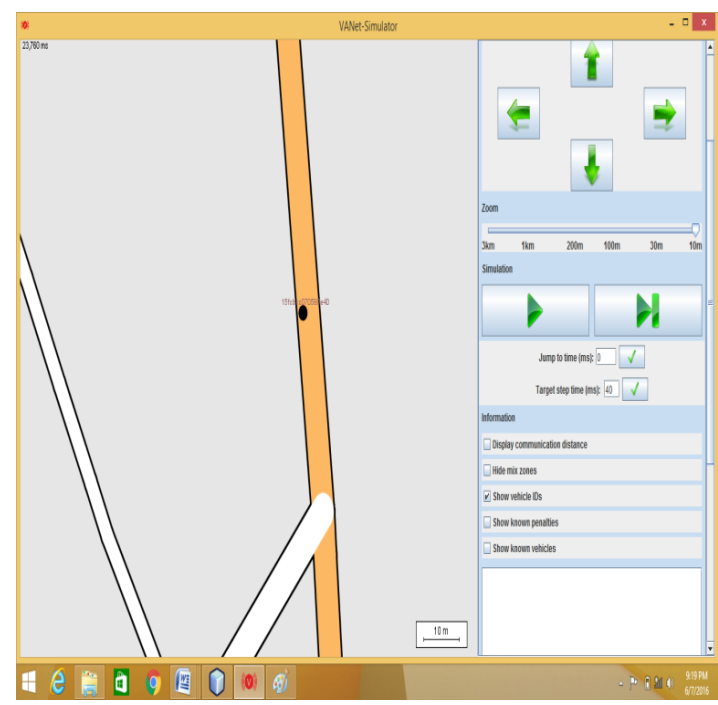

Figure 11: Shows Vehicle ID

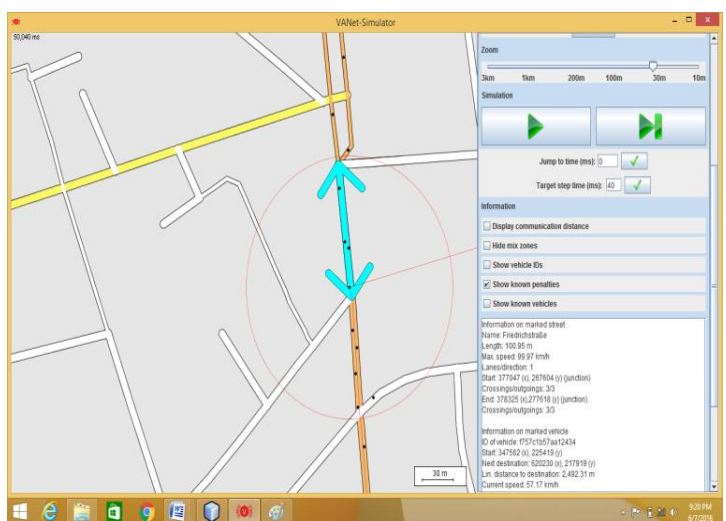

Figure 12: Shows Penalty(i.e Number of Accident in that Lane)

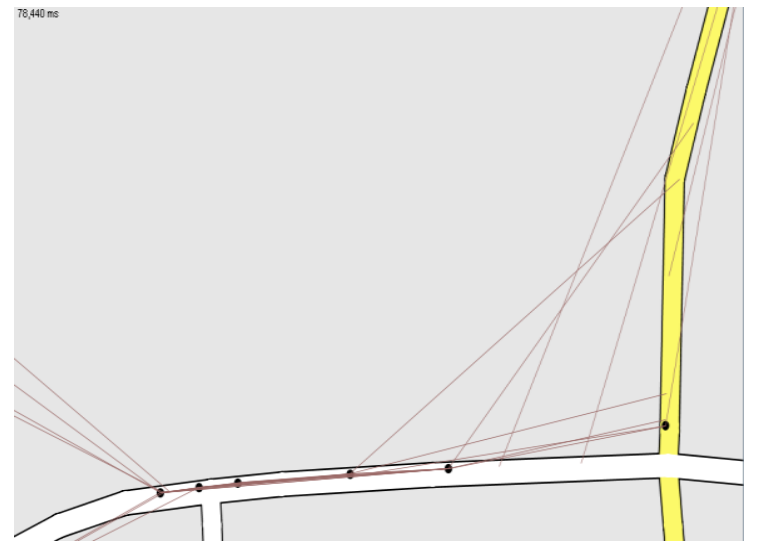

Figure 13: Shows a Known Vehicle

\section{CONCLUSION}

In this paper consists a simulated vehicle to vehicle communication to avoid accidents. This is very useful in heavy traffic and place where lots of accidents happen. This provides communication with other vehicles, distance between the vehicles, and number of vehicles in the communicating range. This is very useful in real time traffic management and is also trustworthy.

\section{REFERENCES}

[1] M. Kavitha, S.S. Tangade and S.S. Manvi, "Distributed trust \& time management strategy in VANETs", Fourth International Conference on Computing, Communications and Networking Technologies (ICCCNT), Pp. 1-6, 2013.

[2] "Trust Computation in VANETs", International Conference on Communication Systems and Network Technologies 2013.

[3] An Efficient Trust Management System for Balancing the Safety and Location Privacy in VANETs, 978-0-7695-4745-9/12, 2012

[4] "Trust Management Schemes in VANET and Countermeasures", IEEE $22^{\text {nd }}$ paper on indoor and mobile communications, 2011.

[5] "RGTE:A Reputation-based Global Trust Establishment in VANETs", International Conference on Intelligent Networking and Collaborative Systems, 2013.

[6] "Establishing Trust in a Vehicular Network", Vehicular networking conference, 2013.

[7] Secure and Revocable Node Authentication in Vehicular Ad-Hoc Networks, 978-1-4799-3755-4/13/\$31.00, 2013.

[8] P. Papadimitratos, L. Buttyan, T. Holczer, E. Schoch, J. Freudiger, M. Raya and J.P. Hubaux, "Secure vehicular communication systems: design and architecture", IEEE Communications Magazine, Vol. 46, No. 11, Pp. 100-109, 2008. 


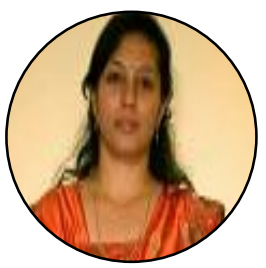

Swathi Shastry, Presently working as Assistant Professor in KLS, VDRIT, Haliyal. She was born on $9^{\text {th }}$ june 1988. Completed her schooling in Presentation Girls High School, Dharwad. She then completed her B. E in KLS VDRIT, Haliyal by 2010. She pursued her masters at VTU Regional Center PG studies, Belgavi by 2014. She joined VDRIT ans Foreman in year 2010. Served for two years and then persued her masters. She joined back as lecturer in 2011 and now serving as Assisitant Professor*. (Email:swathishastry9688@gmail.com)

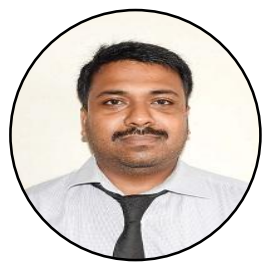

T.R. Naveenkumar, presently working as Assistant Professor in KLS, VDRIT, Haliyal. He was born on 21/11/1985. Completed his schooling at V. S. Pillay, Hubli. He pursued his B. E in BEC, Bagalkot in the year 2009. He pursued his $M$. Tech in CSE at NMAMIT, Nitte. He joined KLS VDRIT Haliyal as lecturer in 2011 and is presently serving as Assistant Professor* in the same college. (Email: trnaveenkumar75@gmail.com) 